\title{
Predicting relapse of Graves' disease following treatment with antithyroid drugs
}

\author{
LIN LIU ${ }^{1}$, HONGWEN LU ${ }^{1}$, YANG LIU ${ }^{2}$, CHANGSHAN LIU ${ }^{1}$ and CHU XUN ${ }^{3}$ \\ ${ }^{1}$ Department of Endocrinology, Weifang People's Hospital, Weifang, Shandong 261041; \\ ${ }^{2}$ Department of Endocrinology, Kailuan General Hospital, Tangshan, Hebei 063000; \\ ${ }^{3}$ Department of Genetics, Shanghai-MOST Key Laboratory of Health and Disease Genomics, \\ Chinese National Human Genome Center, Shanghai Academy of Science and Technology,
} Shanghai 201203, P.R. China

Received November 5, 2014; Accepted December 4, 2015

DOI: $10.3892 /$ etm.2016.3058

\begin{abstract}
The aim of the present study was to monitor long term antithyroid drug treatments and to identify prognostic factors for Graves' disease (GD). A total of 306 patients with GD who were referred to the Endocrinology Clinic at Weifang People's Hospital (Weifang, China) between August 2005 and June 2009 and treated with methimazole were included in the present study. Following treatment, patients were divided into non-remission, including recurrence and constant treatment subgroups, and remission groups. Various prognosis factors were analyzed and compared, including: Patient age, gender, size of thyroid prior to and following treatment, thyroid hormone levels, disease relapse, hypothyroidism and drug side-effects, and states of thyrotropin suppression were observed at 3, 6 and 12 months post-treatment. Sixty-five patients $(21.2 \%)$ were male, and 241 patients $(78.8 \%)$ were female. The mean age was $42 \pm 11$ years, and the follow-up was $31.5 \pm 6.8$ months. Following long-term treatment, 141 patients (46\%) demonstrated remission of hyperthyroidism with a mean duration of $18.7 \pm 1.9$ months. The average age at diagnosis was $45.6 \pm 10.3$ years in the remission group, as compared with $36.4 \pm 8.8$ years in the non-remission group $(\mathrm{t}=3.152$; $\mathrm{P}=0.002)$. Free thyroxine (FT)3 levels were demonstrated to be $25.2 \pm 8.9$ and $18.7 \pm 9.4 \mathrm{pmol} / \mathrm{l}$ in the non-remission and remission groups, respectively $(\mathrm{t}=3.326, \mathrm{P}=0.001)$. The FT3/FT4 ratio and thyrotrophin receptor antibody (TRAb) levels were both significantly higher in the non-remission group ( $\mathrm{t}=3.331,3.389, \mathrm{P}=0.001)$, as compared with the remission group. Logistic regression analysis demonstrated that
\end{abstract}

Correspondence to: Dr Chu Xun, Department of Genetics, Shanghai-MOST Key Laboratory of Health and Disease Genomics, Chinese National Human Genome Center, Shanghai Academy of Science and Technology, 250 Bibo Road, Shanghai 201203, P.R. China

E-mail: chux@chgc.sh.cn

Key words: Graves' disease, recurrence, antithyroid agents, prognostic factors elevated thyroid size, FT3/FT4 ratio and TRAb at diagnosis were associated with poor outcomes. The ratio of continued thyrotropin suppression in the recurrent subgroup was significantly increased, as compared with the remission group $(\mathrm{P}=0.001)$, as thyroid function reached euthyroid state at 3, 6 and 12 months post-treatment. Patients with GD exhibiting large thyroids, high pre-mediation TRAb levels and elevated FT3/FT4 ratios responded less markedly to antithyroid drug treatments, as compared with patients not exhibiting these prognostic factors. Furthermore, patients with large thyroids, post-medication ophthalmopathy and continued thyrotropin suppression demonstrated higher rates of recurrence.

\section{Introduction}

Graves' disease (GD) is among the most prevalent organ-specific autoimmune diseases and is the most common cause of hyperthyroidism worldwide, accounting for $60-80 \%$ of all thyrotoxicosis cases (1). Current therapeutic strategies for the treatment of patients with GD include antithyroid drugs (ATD), thyroid ablation with radioiodine and surgery. In China and Europe, conservative therapy with ATD is the first-line therapy (2). Methimazole (MMI) is the most commonly used ATD for long-term treatments of 12-18 months $(3,4)$; however, the rate of relapse following ATD therapy is $40-50 \%$, and numerous patients require further treatment (3).

The ability of thionamide therapy to directly affect the immunological outcomes of GD has been supported by previous in vitro and in vivo experimental evidence (5). The antithyroid drug MMI predominantly appears to affect B cells and possibly accessory cell function (6). MMI is suitable for use in children and adolescents with GD due to its inhibition of thyroid hormone synthesis, despite the risk of major adverse reactions (7). Unfavorable outcomes are typically due to side-effects, including gastrointestinal upset, facial excoriation, thrombocytopenia, neutropenia and liver enzyme elevation; warfarin-associated coagulopathy and myasthenia gravis have also been associated with MMI treatment, but are rare (8).

A previous study has revealed that MMI dose, pretreatment serum T3 levels and goitre size are the major determinants 
of the therapeutic response of GD patients to MMI (9). By analyzing the factors affecting GD relapse, the efficacy of ATD as a treatment for GD and the probability of recurrence of GD hyperthyroidism following the withdrawal of ATD therapy, therapeutic strategies for the treatment of GD may be improved.

The present study was conducted in order to observe the relapse rate of patients with GD receiving long-term treatment with ATD and to evaluate the factors that affect the relapse of hyperthyroidism following the withdrawal of antithyroid therapy.

\section{Materials and methods}

Study protocol. The present study protocol was approved by the Ethics Committee and Health Authorities of Weifang People's Hospital (Weifang, China) according to their regulations. Written informed consent was obtained from all subjects prior to the initiation of the study.

Patients. The present study investigated patients with newly-diagnosed GD who were referred to the Endocrinology Clinic at Weifang People's Hospital between August 2005 and June 2009. A total of 306 eligible Chinese patients with GD were enrolled and treated with antithyroid drugs, including MMI.

Patients included 65 males and 241 females aged $19-61$ years (mean age, $42 \pm 11$ years) who were diagnosed based on the following commonly accepted clinical and laboratory criteria (10): Hyperthyroidism, diffuse goiter without nodular formation as detected by ultrasound and serum-positive for thyrotrophin receptor antibody (TRAb). Diagnosis of hyperthyroidism was based on the symptoms of thyrotoxicosis and elevated free thyroxine (FT) 4 levels with low thyroid stimulating hormone (TSH). The goiter was classified into three grades according to the World Health Organization classification of goiter (11). The NOSPECS classification was used to grade the changes in thyroid-associated orbitopathy (referred to in the present study as eye syndrome) (12).

Patients were excluded based on the following criteria: Allergy or other side effects of ATD; alanine aminotransferase (ALT) or aspartate aminotransferase (AST) levels two times higher than the normal upper range (ALT, $40 \mathrm{IU} / \mathrm{l}$ and AST, $40 \mathrm{IU} / 1)$; non-compliant due to a psychiatric or other serious disease; could not complete long-term treatment due to a lack of response to previous treatment, thus were treated with other methods, including radioactive iodine or surgery. Patients who did not complete the follow-up course were also excluded.

Study design. All patients were administered MMI daily (Merck Millipore, Darmstadt, Germany) at a starting dose of 10-30 mg with follow-up visits at 2 weeks, followed by once every month and a titration regimen for $\sim 18$ months. Medical and laboratory assessments of FT3, FT4, TSH and total white blood cell counts were performed at each visit. ALT, AST and TRAb levels and thyroid volume were measured at 3-month intervals. If euthyroidism was achieved, which was defined as the elimination of the majority of hyperthyroidism symptoms and restored serum levels of TSH and FT4, pharmacological therapy was gradually adjusted and a continued dose of
MMI was administered in order to maintain euthyroidism. If subclinical hypothyroidism was detected, which is defined as serum TSH levels $>10 \mathrm{mU} / 1$ and simultaneous normal serum FT4 levels, L-thyroxine (L-T4) was administered and dosage was gradually adjusted until euthyroidism was achieved. At the same time, L-T4 was also administered for exophthalmos deterioration.

Following the withdrawal of antithyroid drugs, patients were followed up for $\geq 2$ years, once every month for the first 6 months followed by once every 3 months. In addition, patients were immediately reviewed if they felt they may be suffering a disease relapse. Each follow-up visit consisted of clinical assessment of thyroid status and measurements of serum FT3, FT4 and TSH levels were collected.

Patients were withdrawn from treatment with antithyroid drugs according to the following criteria: i) Patients received MMI for $\sim 18$ months; ii) euthyroidism was achieved for $>6$ months with the lowest daily dose of $5 \mathrm{mg}$ MMI; iii) TRAb was converted to negative, as TRAb levels reduced to normal.

Remission was defined as the state when euthyroidism, demonstrated by normal ranges of FT3, FT4 and TSH levels, was achieved following the withdrawal of antithyroid drugs for $>6$ months. Relapse was defined as the state when hyperthyroidism was detected at any time following treatment with ATD. Non-remission was defined when recurrence occurred following the termination of ATD treatment, even if the patients received ATD treatment for $>2$ years.

Patients who experienced relapse of hyperthyroidism following ATD treatment cessation, or those who did not complete long-term treatment due to a lack of response to ATDs and were treated with other methods, such as radioactive iodine or surgery, were considered to be relapsed cases. All patients were monitored and followed up by endocrinologists. A patient record was completed for each patient, including age, gender, initial treatment time, duration of treatment, types of ATDs administered, estimated volume of thyroid, relapse of disease status during and after treatment, duration of follow-up course serum TSH, FT3, and FT4 levels, and TRAb levels at the initiation and termination of treatment.

Laboratory measurements. Blood cells were quantified using a Coulter GEN-S System 2 analyzer (Beckman Coulter, Inc., Brea, CA, USA). Agranulocytosis was defined as a total granulocyte count of $<110$ granulocytes per liter of blood. Serum TSH (reference range, 0.42-4.60 IU/ml) and FT4 (reference range, 10.61-25.22 pmmol/l) levels were measured using a chemiluminescence assay (Unicel DxI 800; Beckman Coulter, Inc.). TRAbs were assessed using a TSH REZAK ${ }^{\circledR}$ radioimmunoassay kit (Medipan Gmbh, Berlin, Germany). TRAb was considered positive at levels $>14 \mathrm{U} / 1$ and with intra- and inter-assay coefficients of variation $<4.8$ and $4.6-7.6 \%$, respectively. Serum ALT and AST levels were determined using enzymatic procedures on an automated clinical chemistry analyzer, according to the manufacturer's protocol (AU2700; Olympus Corporation, Tokyo, Japan). Thyroid volume was estimated via ultrasonography using a $7.5-\mathrm{MHz}$ linear array transducer (GE Healthcare Bio-Systems, Milwaukee, WI, USA). All examinations were performed and interpreted by the same experienced radiologist. Thyroid volume values 
Table I. Baseline characteristics of patients with Graves' disease prior to treatment with methimazole.

\begin{tabular}{lccc}
\hline Characteristic & Remission group $(\mathrm{n}=141)$ & Non-remission group $(\mathrm{n}=165)$ & P-value \\
\hline Age (years) & $45.6 \pm 10.3$ & $36.4 \pm 8.8$ & 0.002 \\
FT3 (pmmol/l) & $18.7 \pm 9.4$ & $25.2 \pm 8.9$ & 0.001 \\
FT4 (pmmol/l) & $39.3 \pm 10.8$ & $40.7 \pm 11.2$ & 0.62 \\
FT3/FT4 ratio & 0.47 & $0.003 \pm 0.002$ & 0.001 \\
sTSH (mU/l) & $0.003 \pm 0.001$ & $29 \pm 12$ & \\
TRAb positive (\%) & $19 \pm 11$ & $65(39.4 \%)$ & 0.426 \\
Eye syndrome & & $100(60.6 \%)$ & \\
Yes & $20(14.2 \%)$ & $71(51.4 \%)$ \\
No & $121(85.8 \%)$ & $153(92.7 \%)$ \\
Goiter & $70(49.6 \%)$ & 0.001 \\
Grade I & $12(7.3 \%)$ & - \\
Grade II and above & & & \\
\hline
\end{tabular}

Eye syndrome was defined by a NOSPECS score $\geq 2$. FT, free thyroxine; sTSH, sensitive thyroid stimulating hormone; TRAb; thyrotrophin receptor antibody.

were obtained by calculating the volumes of both lobes as follows: Lobe $(\mathrm{ml})=$ length $\mathrm{x}$ width $\mathrm{x}$ depth $(\mathrm{mm}) \times 0.479$. Nodules and/or cystic areas were included in the thyroid volume (reference values: Females, $18 \mathrm{ml}$; males, $25 \mathrm{ml}$ ).

Statistical analysis. SPSS software (version 16.0; SPSS, Inc., Chicago, IL, USA) was used for data collection and $\chi^{2}$ test, and analysis of variance was used for analysis. $\mathrm{P}<0.05$ was considered to indicate a statistically significant difference.

\section{Results}

Baseline patient characteristics. The baseline clinical characteristics of the patients are presented as proportions in Table I. The baseline data of the two groups demonstrated that patients in the non-remission group tended to be younger at the age of onset and have larger thyroid glands, more notable thyroid-associated ophthalmopathy and increased FT3 levels $(\mathrm{P}=0.001)$, FT3/FT4 ratio $(\mathrm{P}=0.001)$ and TRAb $(\mathrm{P}=0.001)$, as compared with the remission group. However, no significant differences in as gender, family history, course of disease and smoking were observed between the two groups.

Post-treatment outcomes. Patients in the recurrence sub-group demonstrated markedly increased thyroid gland volumes and were more likely to develop thyroid associated ophthalmopathy, as compared with those in the remission group $(\mathrm{P}<0.05$; Table II). Increased incidence of GD recurrence was demonstrated in patients with larger thyroid glands and ophthalomopathies at the time of drug withdrawal. The incidence of notably enlarged thyroid glands and/or thyroid associated ophthalmopathy was higher in the constant treatment sub-group, as compared with those in the other two groups, and the levels of serum FT3, FT3/FT4, sensitive (s)TSH differed significantly between the constant treatment group and the two other groups $(\mathrm{P}<0.05)$.
Logistic regression analysis demonstrated that the following factors significantly affected drug treatment outcomes: Thyroid size [OR, 8.725; 95\% confidence interval (CI), 4.103-30.519; $\mathrm{P}=0.001]$; TRAb level (OR, 1.712; 95\% CI, 1.042-2.116; $\mathrm{P}=0.001)$; and the FT3/FT4 ratio $(\mathrm{OR}$, 1.629; 95\% CI, 1.104-2.654; $\mathrm{P}=0.023$ ).

sTSH levels were compared between the patients in the recurrence and remission groups who had normal thyroid hormone levels at 3,6 and 12 months following drug withdrawal. The percentage of cases with sTSH suppression, defined as below the normal lower limit, were $101(94.4 \%)$, $80(74.8 \%)$ and $12(8.5 \%)$ in the recurrence group at 3, 6 and 12 months after drug withdrawal, respectively, as compared with $58(41.1 \%), 12(8.5 \%)$ and $0(0 \%)$, respectively, in the remission group. The differences between the two groups were statistically significant at each time point $(\mathrm{P}<0.01)$, indicating that the time required for the restoration of sTSH concentration during treatments may serve as an evaluation index for the efficacy of ATD treatment. In patients with delayed STSH restoration, the efficacy of ATD was poor and the incidence of recurrence was high.

\section{Discussion}

ATD therapy remains the primary method of treatment for GD-induced hyperthyroidism in various countries. However, the relapse rate is high following the cessation of ATD treatment (3). ATD prognosis factors have previously been studied (13); however, a unified classification criteria for the prognosis of treated hyperthyroidism for remission, cure, drug withdrawal and recurrence is yet to be established worldwide. In China, the criteria outlined by Bai (14) are most commonly adopted. In the present study, the rate of relapse following treatment with ATD was $53.9 \%$ after 31.5 \pm 6.8 -month follow-up. In a meta-analysis of GD performed by Bai (14), the relapse rate decreased with age, 
Table II. Comparison between the remission and non-remission groups following treatment with methimazole.

\begin{tabular}{|c|c|c|c|c|c|}
\hline \multirow[b]{2}{*}{ Characteristic } & \multirow[b]{2}{*}{ Remission group } & \multicolumn{2}{|c|}{ Non-remission groups } & \multirow[b]{2}{*}{$\mathrm{P}_{1}$} & \multirow[b]{2}{*}{$\mathrm{P}_{2}$} \\
\hline & & Recurrence & Constant treatment & & \\
\hline $\mathrm{N}$ & 141 & 107 & 58 & - & - \\
\hline Time of therapy & $18.7 \pm 1.9$ & $20.9 \pm 2.7$ & $26.2 \pm 2.3$ & 0.024 & 0.009 \\
\hline Eye syndrome & & & & 0.004 & $<0.001$ \\
\hline Yes & $17(12.1 \%)$ & $28(26.2 \%)$ & $26(44.8 \%)$ & & \\
\hline No & $124(87.9 \%)$ & $79(73.8 \%)$ & $32(55.2 \%)$ & & \\
\hline Goiter & & & & $<0.001$ & $<0.001$ \\
\hline Grade I & $100(70.9 \%)$ & $27(25.2 \%)$ & $11(19.0 \%)$ & & \\
\hline Grade II and above & $41(29.1 \%)$ & $80(74.8 \%)$ & $47(81.1 \%)$ & & \\
\hline FT3 $(\mathrm{pmmol} / \mathrm{l})$ & $3.7 \pm 1.0$ & $4.1 \pm 0.8$ & $4.3 \pm 1.0$ & 0.087 & 0.136 \\
\hline FT4 (pmmol/l) & $13.7 \pm 1.3$ & $14.2 \pm 1.5$ & $14.6 \pm 1.8$ & 0.401 & 0.458 \\
\hline sTSH (mIU/l) & $2.061 \pm 1.089$ & $1.961 \pm 0.983$ & $0.370 \pm 0.059$ & 0.536 & 0.002 \\
\hline TRAb & $9 \pm 3$ & $11 \pm 5$ & $12 \pm 4$ & 0.053 & 0.241 \\
\hline
\end{tabular}

$\mathrm{P}_{1}$, recurrence group vs. remission group; $\mathrm{P}_{2}$, recurrence group vs. constant treatment group; $\mathrm{P}_{3}$, recurrence group vs. constant treatment group for eye syndrome and goiter; FT, free thyroxine; sTSH, sensitive thyroid stimulating hormone; TRAb, thyrotrophin receptor antibody.

the rate of relapse was $47 \%$ in patients $>40$ years old and $76 \%$ in patients $<20$ years old.

Among the 306 patients with GD assessed in the present study, $165(53.9 \%)$ patients were not responsive to the treatment and $141(46.1 \%)$ demonstrated remission following treatment with ATD; however, disease recurrence was detected during the 2-year follow-up period after drug withdrawal. Patients in the non-remission group were younger at the age of disease onset $(\mathrm{P}<0.002)$ and demonstrated increased FT3 levels $(\mathrm{P}<0.001)$, higher $\mathrm{FT} 3 / \mathrm{FT} 4$ ratios $(\mathrm{P}=0.001)$ and increased levels of TRAb $(\mathrm{P}=0.001)$ at the onset of disease, as compared with those in the remission group. Logistic regression analysis demonstrated that TRAb levels, the size of enlarged thyroid glands and the FT3/FT4 ratio at the onset of disease were independent factors affecting treatment outcomes.

GD is an organ-specific autoimmune disease of the thyroid caused by TRAb directing against the TSH receptor in the thyroid follicular cells (15). Treatment outcomes of ATD administration may be predicted via TRAb levels at the time of drug withdrawal. In the present study, a number of patients with GD achieved a long-term remission following ATD therapy, which may be a result of the direct immunosuppressive action of ATDs (16). The relapse rate was high in those with positive TRAb at the time of drug withdrawal (17).

Vitti et al (18) demonstrated that high relapse rates occurred in patients with high TRAb levels at the onset of hyperthyroidism, which is consistent with the results of the present study.

A previous study has also demonstrated that high remission rates and long-term remission were more prevalent among patients that presented with smaller goiters at the onset of hyperthyroidism or significantly diminished goiters following treatment (19). Furthermore, Cooper (16) also demonstrated that patients with significantly enlarged thyroid glands at the initial stage and a high serum T3/T4 ratio following the drug withdrawal exhibited high recurrence rates.
Following 5 years of follow-up of GD patients with various goiter sizes at the onset of disease, Laurberg (20) found that remission rates were higher in patients with normal or mild goiter sizes prior to treatment, as compared with patients with moderately enlarged glands. Furthermore, Manji et al (21) demonstrated that patients with enlarged thyroid glands at the initial stage tended to develop severe GD.

The present study demonstrated that a high relapse rate following ATD therapy was significantly associated with goiter size at the onset of disease and enlarged glands at the time of drug withdrawal. Furthermore, it was also demonstrated that the FT3/FT4 ratio at the onset of GD was elevated in the non-remission group, as compared with the remission group, which is consistent with the study conducted by Yoshimura et al (22), which suggested that the ratio may be used as an index to predict treatment outcomes of hyperthyroidism following ATD therapy.

Previous studies have demonstrated that smoking is associated with the occurrence, development and prognosis of GD $(23,24)$. However, the results of the present study demonstrated that various relapse parameters $(25,26)$, including gender, disease course, family history of thyroid diseases and smoking, did not significantly affect disease regression rates. Therefore, these parameters may not be used to predict the outcome of drug treatment for GD. Due to the high percentage (78.8\%) of female patients included in the present study there were only 5 cases of smokers, as traditionally, women do not smoke in China; therefore, no clear association between smoking and GD was observed, in contrast with a previous study (24). Therefore, future large cohort studies with more smokers are required in order to investigate the association between GD and smoking.

Negative feedback inhibition is the primary regulatory mechanism exhibited by thyroid hormone on TSH (27). Following treatment with ATD, sTSH levels in hyperthyroidism patients should return to normal with the 
normalization of thyroid hormone. However, certain patients are subjected to sustained suppression of sTSH in spite of the normalization of thyroid hormone (20). These patients usually require prolonged medical treatment and often suffer from high recurrence rates. In the past, due to the lack of sensitive detection methods, sTSH was seldom used as a clinical evaluation index of sub-clinical hyperthyroidism and had limited use in the evaluation of hyperthyroidism recurrence $(28,29)$. However, with the application of highly sensitive detection technologies, the clinical value of TSH levels has increased, particularly in predicting the recurrence of hyperthyroidism and evaluating sub-clinical hypothyroidism and hyperthyroidism (30).

The duration of therapy that is necessary for individual patients with GD remains unclear (31). Previous studies have demonstrated that treatment durations of $>18$ months were not able to improve remission rates (32). Furthermore, Quadbeck et al (33) demonstrated that TSH suppression at the time of drug withdrawal was a predicting factor for the recurrence of GD. In the present study, the number of patients with normal sTSH level was reduced in the non-remission group, as compared with the remission group at 3,6 and 12 months following ATD treatments when euthyroidism was achieved.

The results of the present study indicated that patients in the non-remission group experienced delayed STSH restoration, and patients demonstrating STSH suppression had a high tendency for GD recurrence if ATG therapy was prematurely terminated. Therefore, patients with low levels of STSH should receive prolonged treatment with ATG until sTSH levels normalize.

Logistic regression analysis failed to identify ophthalmopathy as a prognostic factor for GD recurrence, although the incidence of ophthalmopathy was significantly increased in the recurrence sub-group, as compared with the remission group $(\mathrm{P}<0.05)$. Following a year of treatment with ATG, the incidence of ophthalmopathy was significantly higher in the constant treatment subgroup, as compared with the remission and recurrence sub-groups $(\mathrm{P}<0.05)$. Regardless, we hypothesize that GD patients who did not show improved symptoms following ATD therapy continued to experience higher recurrence rates of ophthalmopathy. Overall, GD patients with large thyroids, high TRAb levels, and high FT3/FT4 ratios at the onset of disease tended to fail to respond to ATD. A limitation of the present study is that the patients enrolled were all northern Chinese, predominantly from the Shandong Peninsula.

In conclusion, patients with large thyroids and ophthalmopathy following treatment with ATD demonstrated increased recurrence rates; and patients with euthyroid and TSH restoration following treatment with ATD were more likely to experience recurrence. Therefore, the results of the present study suggested that patients with GD who present with the above characteristics should be provided with alternative treatment.

\section{Acknowledgements}

The present study was supported by grants from the Ministry of Health (grant no. 201202008) and the Shandong Province Health Department (grant no. 2005JW0033).

\section{References}

1. Jameson JL and Weetman AP: Disorders of thyroid gland. In: Harrison's Principles of Internal Medicine. Kasper DL, Braunwald E, Fauci AS, Hauser SL, Longo DL, Jameson JL (eds). 16th edition. McGraw-Hill, New York, NY, pp2104-2127, 2005.

2. Wille T, Müller B, Noth D, Bürgi U and Diem P: Long-term follow up after antithyroid drug treatment in Graves' disease. Praxis (Bern) 1994: 1121-1127, 2006 (In German).

3. Abraham P, Avenell A, Park CM, Watson WA and Bevan JS: A systematic review of drug therapy for Graves' hyperthyroidism. Eur J Endocrinol 153: 489-498, 2005.

4. Weetman AP: Graves' disease. N Engl J Med 343: 1236-1248, 2000.

5. Bagnasco M, Venuti D, Caria M, Pizzamo G, Ferrini O and Canonica GW: Methimazole, $\gamma$-'Interferon and Graves' Disease. In: Thyroid Autoimmunity. Pinchera A, Ingbar SH, McKenzie JM and Fenzi GF (eds). 1st Edition. Springer US, New York, NY, pp445-447, 1987.

6. Bagnasco M, Venuti D, Ciprandi G, Pesce G, Paolieri F and Canonica GW: The effect of methimazole on the immune system is unlikely to operate directly on T lymphocytes. J Endocrinol Invest 13: 493-499, 1990.

7. Sato H, Minagawa M, Sasaki N, Sugihara S, Kazukawa I, Minamitani K, Wataki K, Konda S, Inomata H, Sanayama K and Kohno Y: Comparison of methimazole and propylthiouracil in the management of children and adolescents with Graves disease: Efficacy and adverse reactions during initial treatment and long-term outcome. J Pediatr Endocrinol Metab 24: 257-263, 2011.

8. Trepanier LA: Medical management of hyperthyroidism. Clin Tech Small Anim Pract 21: 22-28, 2006.

9. Benker G, Vitti P, Kahaly G, Raue F, Tegler L, Hirche H and Reinwein D: The European Multicenter Study Group: Response to methimazole in Graves' disease. Clin Endocrinol (Oxf) 43: 257-263, 1995.

10. Chinese Society of Endocrinology: Guidelines for diagnosis and treatment of thyroid diseases in China: Hyperthyroidism. Chi J Intern Med 46: 876-882, 2007.

11. World Health Organization, International Council for Control of Iodine Deficiency Disorders and UNICEF: Indicators for assessing Iodine Deficiency Disorders and their control through salt iodization. 1st edition. World Health Organization, Geneva, Switzerland, pp5-8, 13-35, 1994.

12. Werner SC: Modification of the classification of the eye changes of Graves' disease. Am J Ophthalmol 83: 725-727, 1977.

13. Shi BY: Antithyroid drugs: Rational and normative application all the more. Zhong Hua Nei Fen Mi Dai Xie Za Zhi 25: 245-246, 2009 (In Chinese).

14. Bai Y (ed): Hyperthyroidism: In: Thyroid disease: Basic and Clinic. 1st edition. Beijing Scientific and Technological Literature Publishing House; Beijing, pp244-278, 2003.

15. Akamizu T, Moriyama K, Miura M, Saijo M, Matsuda F and Nakao K: Characterization of recombinant monoclonal antithyrotropin receptor antibodies (TSHRAbs) derived from lymphocytes of patients with Graves' disease: Epitope and binding study of two stimulatory TSHRAbs. Endocrinology 140: 1594-1601, 1999.

16. Cooper DS: Antithyroid drugs. N Engl J Med 352: 905-917, 2005.

17. Lan L, Teng WP, Shi BY, Liu XJ, Li H, Liu J, Wu XY, Xu L and Wang FX: Related factors in predicting relapse of Graves disease after treatment with antithyroid drug. Zhong Hua Nei Fen Mi Dai Xie Za Zhi 25: 247-249, 2009 (In Chinese).

18. Vitti P, Rago T, Chiovato L, Pallini S, Santini F, Fiore E, Rocchi R, Martino E and Pinchera A: Clinical features of patients with Graves' disease undergoing remission after antithyroid drug treatment. Thyroid 7: 369-375, 1997.

19. Wang PW, Liu RT, Tung SC, Chien WY, Lu YC, Chen CH, Kuo MC, Hsieh JR and Wang ST: Outcome of Graves' disease after antithyroid drug treatment in Taiwan. J Formos Med Assoc 97: 619-625, 1998.

20. Laurberg P: Remission of Graves' disease during anti-thyroid drug therapy. Time to reconsider the mechanism? Eur J Endocrinol 155: 783-786, 2006.

21. Manji N, Carr-Smith JD, Boelaert K, Allahabadia A, Armitage M, Chatterjee VK, Lazarus JH, Pearce SH, Vaidya B, Gough SC and Franklyn JA: Influences of age, gender, smoking and family history on autoimmune thyroid disease phenotype. J Clin Endocrinol Metab 91: 4873-4880, 2006. 
22. Yoshimura Noh J, Momotani N, Fukada S, Ito K, Miyauchi A and Amino N: Ratio of serum free triiodothyronine to free thyroxine in Graves' hyperthyroidism and thyrotoxicosis caused by painless thyroiditis. Endocr J 52: 537-542, 2005.

23. Holm IA, Manson JE, Michels KB, Alexander EK, Willett WC and Utiger RD: Smoking and other lifestyle factors and the risk of Graves' hyperthyroidism. Arch Intern Med 165: 1606-1611, 2005.

24. Prummel MF and Wiersinga WM: Smoking and risk of Graves disease. JAMA 269: 479-482, 1993.

25. Nordyke RA, Gilbert FI Jr and Harada AS: Graves' disease. Influence of age on clinical findings. Arch Intern Med 148: 626-631, 1988

26. Aizawa T, Ishihara M, Hashizume K, Takasu N and Yamada T: Age-related changes of thyroid function and immunologic abnormalities in patients with hyperthyroidism due to Graves' disease. J Am Geriatr Soc 37: 944-948, 1989.

27. Kelly GS: Peripheral metabolism of thyroid hormones: A review. Altern Med Rev 5: 306-333, 2000.

28. Morimoto $\mathrm{K}$ and Inouye K: A sensitive enzyme immunoassay of human thyroid-stimulating hormone (TSH) using bispecific $\mathrm{F}\left(\mathrm{ab}^{\prime}\right) 2$ fragments recognizing polymerized alkaline phosphatase and TSH. J Immunol Methods 205: 81-90, 1997.
29. Perrin A, Theretz A and Mandrand B: Thyroid stimulating hormone assays based on the detection of gold conjugates by scanning force microscopy. Anal Biochem 256: 200-206, 1998.

30. Lu N, Dai P, Gao A, Valiaho J, Kallio P, Wang Y and Li T: Label-free and rapid electrical detection of hTSH with CMOS-compatible silicon nanowire transistor arrays. ACS Appl Mater Interfaces 6: 20378-20384, 2014.

31. Quadbeck B, Janssen OE and Mann K: Problems and new developments in the management of Graves' disease: Medical therapy. Z Arztl Fortbild Qualitatssich 98 (Suppl 5): 37-44, 2004 (In German).

32. Maugendre D, Gatel A, Campion L, Massart C, Guilhem I, Lorcy Y, Lescouarch J, Herry JY and Allannic H: Antithyroid drugs and Graves' disease - prospective randomized assessment of long term treatment. Clin Endocrinol (Oxf) 50: 127-132, 1999.

33. Quadbeck B, Roggenbuck U, Janssen OE, Hahn S, Mann K and Hoermann R; Basedow Study Group: Impact of smoking on the course of Graves' disease after withdrawal of antithyroid drugs. Exp Clin Endocrinol Diabetes 114: 406-411, 2006. 